\title{
Application of big data technology in clinical medicine and scientific research
}

\author{
Li zhiyi $^{1 *}$, Pan Ling ${ }^{1}$, Cui Lifang ${ }^{2}$ and Xie shiping ${ }^{1}$ \\ ${ }^{1}$ Henan University of Chinese Medicine, Zhengzhou, China \\ 2 Henan Province Chinese medicine research institute, Zhengzhou, China \\ *zhiyi102@sina.com
}

\begin{abstract}
With the development of science and technology, big data analysis can process massive amounts data generated by the medical and health industry. Timely acquisition, efficient treatment, rational application of big data, which can provide the reference for clinical diagnosis and treatment of common diseases, provide the basis for the fine management of hospital, provides the platform for personalized health care, and provide data for clinical research. This can be better for clinical medicine, health management and medical research work.
\end{abstract}

Keywords: big data, clinical medicine, scientific research, technology

\section{Introduction}

With the development of science and technology, the ability of human to deal with data is constantly improving. Big data has become a new research area. People can store, develop, utilize and analyze data scientifically, and get more accurate and effective results. Big data technology is known as one of the three major technological changes to lead the prosperity of the future.

This provides us with a new way of looking at the world. Through big data analysis, mining a small amount of data can not be extracted valuable information, service in the economic and social development, which is no longer entirely dependent on random sampling, no longer keen on the pursuit of precision, no longer interested in finding the cause and effect relationship [1]. Analysis of big data in e-commerce, social networking, consulting services, retail, finance, communications, environmental science and so on has produced enormous social value and industrial space, which will also bring revolutionary impact on clinical medicine and scientific research.

\section{What is the medical big data?}

Big data, also known as mega data, refers to the amount of data, the artificial can not be intercepted within a reasonable time, management, processing, and be able to interpreted the information that man can understand. Big data is a data set which is composed of huge quantity, complex structure and many types of data. It is a data processing and application mode based on cloud computing. Through the integration and sharing of the data, the intelligence resources and knowledge service ability are formed by cross multiplex. Data generated by the medical and health industry is a typical big data, with greater capacity, faster generation rate, higher diversity, more value [2].

\section{Development of medical big data}

With the deepening of health care reform, health statistics and information construction technology has been further strengthened. Statistical methods have been improved, variety of information systems are widely used, which lead to the rapid expansion of medical and health data and a geometric progression in geometric progression.

Including the use of image data, medical records, inspection results, medical expenses, etc., all kinds of data, build reasonable advanced data services platform, will be able to for the majority of patients and medical staff, researchers and government decision-making to provide services and assistance [3]. We can get more valuable information by analyzing the large amount of data available. However, due 
to the traditional data center can only focus on the analysis of a single data set, it will result in the separation between different types of data. The big data analysis can aggregate multiple data sets, and focus on the integration of different types of data and correlation analysis.

By the end of 2010, the former ministry of health of China completed the 12th Five Year Plan for health information construction project planning, which identified the construction of China's health information road map. Namely, the construction of national, provincial and municipal health information platform, strengthen public health, medical service, new rural cooperative medical system, the basic drug system, integrated management and other business applications, construction of health records and electronic medical records based databases and one private networks [4]. The platform will form a large-scale, sub regional medical information system, to save the massive medical and health data. How to analyze and use the data more effectively, to provide services and assistance to the majority of patients, medical personnel, scientific research personnel and management personnel, has become an important direction of information technology in the future.

\section{The application prospect of medical big data}

\subsection{To provide reference for clinical diagnosis and treatment of common diseases}

Sometimes experts also cannot meet the clinical diagnosis and treatment of the right difficult miscellaneous diseases [5]. The big data technology through the accumulation of massive medical data mining analysis, on this basis to repeat inspection suggested that treatment of safety warning, drug allergy alert, curative effect evaluation, intelligent analysis diagnosis and treatment plan, predict disease progression and a series of intelligent human-computer interaction applications. This will be able to provide scientific decision-making reference for clinicians to improve the level of clinical diagnosis and treatment, the formation of clinical decision support system from clinical, regression clinical.

Through the large data analysis of the patient data, you can determine which people are susceptible to a certain class of diseases, so that they receive preventive intervention as early as possible. These methods can also help patients choose appropriate treatment options [4]. In addition, the clinical decision support system can also make the doctors from the time-consuming of the simple consultation work freed, so as to greatly improve the efficiency of its diagnosis and treatment.

\subsection{To provide basis for the fine management of hospital}

Fine management is an inevitable requirement of scientific management, and it is a technical way for managers to adjust the product, service and operation process. It is based on the standardization of the premise, the system is to ensure that the data as the standard, information technology as the means, so as to obtain more efficient, more effective and more competitive. Data analysis can be based on large data analysis platform from the hospital outpatient amount, the amount of operation, the number of patients, the utilization rate of beds, bed turnover rate, equipment utilization rate, equipment depreciation rate, the disease spectrum, the patient distribution region, the financial revenue and expenditure, etc.. The current data can be compared with the same period data, preliminary data analysis. Through the comparative analysis of the local similar hospital data, we can find out the cause of the hospital economic operation quality and the gap, seize the weak links in their own work, and take effective measures to improve the quality of the hospital economic operation [6].

\subsection{To provide a platform for individual medical care}

Individual health care is based on a large amount of information from each patient. Through the comprehensive analysis of the characteristics of each patient's pathology, physiology and so on, we can develop a unique, optimal treatment and prevention program for each patient, which can improve the pertinence of the treatment to achieve the best effect efficacy [7]. Individual health care needs 
comprehensive analysis of all aspects of the patient's information, while requiring the processing of such a big data approach and the ability. The development of technology makes the information of patients in modern medical routine collection is very huge, and the ability to analyze information also has a great improvement, so that individual health care becomes possible [8].

\subsection{To provide data for clinical research}

Life science research is facing the transformation from the experimental driven to the data driven. The emergence of big data has led to a new scientific research model. That is the scientific researcher only need to find the information, knowledge and intelligence directly from the massive data, even without direct contact with the object to be studied [9]. In 2007, late of the Turing Award winner Jim gray depicts the data intensive scientific research the Fourth Paradigm in his last speech [10]. He put big data research from the Third Paradigm separated alone as a kind of research paradigm, that the Fourth Paradigm Paradigm may be the only way to solve the system we are faced with some of the most difficult global challenges. In the process of scientific research, the use of big data can subvert many of our previous research results, for us to bring unexpected benefits.Health data resources for the medical and health industry, to promote the development of medical and health technology to provide the conditions for big data. The relevant standards and software applications of clinical medical big data are still in the initial stage now, which can be used to open up a lot of space, providing an unprecedented opportunity. But the researchers are faced with how to get big data and field, and effectively deal with big data, the reasonable use of the real problem of big data. We need to explore new ways, technologies and methods on the basis of cross penetration of multi subjects, to serve the clinical medicine, health management and medical scientific research.

\section{Reference}

[1] Zheng Lingwei. Step into the age of big data. Informatization construction, 2013, (1): 10-13

[2] Gao Hansong, Xiao Ling, Xu Dewei, Janson, et al. Journal of Medical Intelligence .2013, 34 (5): $7-12$

[3] Zhou Guanghua, Xin Ying, Zhang Yajie, et al. Study on the application of large data in medical and health field. Chinese Journal of Health Informatics and Management, 2013, 10 (4): 296-300

[4] Liu Xiaoliang, Wang Kun, Ma Jun. Thinking about the construction of health information in the era of big data . Chinese Journal of Health Informatics and Management, 2014, 11 (1): 43-46

[5] Jian Yunjiang, Yang Yan, Liu Yaqi. The application of big data in medicine. Medicine and Pharmacy of Yunnan,2015, 36 (6): 656-659

[6] Li Ping, Liu Peng. Thinking of strengthening the management of hospital fine management . Modern Hospital Management, 2011, 5: 28-30

[7] Ginsburg G S,Willard H F. Genomic and personalized medicine:foundations and applications. Transl Res, 2009, 154 (6): 277-287

[8] Li Jing, Gu Jiang. Opportunities and challenges in the era of personalized medicine and big data. Medicine \& Philosophy, 2014, 35 (1A): 5-11

[9] Li Guojie, Cheng Xueqi . Big data research: the major strategic areas of science and technology and economic and social development -- Research Status and scientific thinking of big data. Bulletin of the Chinese Academy of Sciences,.2012, 27 (6): 647-656 
[10] Tony Hey, Stewart Tansley, Kristin Tolle. The Fourth Paradigm: Data-Intensive Scientific Discovery. Microsoft, 2009, October 16 